\title{
DETECTION OF THE NUMBER OF SIGNALS USING A MULTIPLE HYPOTHESIS TEST
}

\author{
Pei-Jung Chung ${ }^{1)}$, Johann F. Böhme ${ }^{2)}$, Alfred O. Hero ${ }^{1)}$, Christoph F. Mecklenbräuker ${ }^{3)}$ \\ ${ }^{1)}$ Dept. of Electrical Engineering and Computer Science \\ University of Michigan, USA \\ ${ }^{2}$ Dept. of Electrical Engineering and Information Science \\ Ruhr-Universität Bochum, Germany \\ ${ }^{3)} \mathrm{ftw}$. Telecommunications Research Center Vienna, Austria \\ peijung@eecs.umich.edu, boehme@sth.ruhr-uni-bochum.de, hero@eecs.umich.edu,cfm@ftw.at
}

\begin{abstract}
This work presents a novel approach to detect multiple signals embedded in noisy observations of a sensor array. We formulate the detection problem as a multiple hypothesis test. To control the global level of the multiple test, we apply the false discovery rate (FDR) criterion recently suggested by Benjamini and Hochberg instead of the classical familywise error rate (FWE) criterion. The proposed method is tested by the simulated data. Results show that the FDRcontrolling procedure is more powerful than the FWE- controlling procedure. The performance improvement is most significant for a large number of signals and low SNRs.
\end{abstract}

\section{INTRODUCTION}

This work discusses signal detection using a multiple hypothesis test. Estimating the number of signals embedded in noisy observations is a key issue in array signal processing, harmonic retrieval, wireless communication and geophysical application.

Methods based on the information theoretic criterion and minimum description length (MDL) criterion were proposed in [8] [9]. In [3][6], a multiple testing procedure was suggested to determine the number of signals. In contrast to the subspace based methods [8] [9], the test statistics in [3][6] are derived from the likelihood ratios (LR). As pointed out in [6], the LR-based approach has a lower signal to noise ratio (SNR) threshold and a better performance in simulation and real data processing.

A major concern in multiple testing problems is the control of type I errors. The detection procedure in [3][6] applied the Bonferroni-Holm procedure [5] to control the global level of the test. The Bonferroni-Holm procedure controls the probability of committing any type I error in families of comparisons under simultaneous consideration. The con- trol of familywise error-rate (FWE) often leads to conservative results. To overcome this drawback, Benjamini and Hochberg suggest that the false discovery rate (FDR) may be the appropriate error rate to control in many applications [1]. The Benjamini-Hochberg procedure was shown to be much more powerful than comparable procedures that control FWE. Motivated by its potential of a gain in power, we apply the Benjamini-Hochberg procedure to our detection scheme.

This paper is outlined as follows. We give a brief description of the signal model in the next section. Then we present the multiple test procedure for signal detection. Section 4 introduces the concept of false discovery rate (FDR) and the Benjamini-Hochberg procedure. Simulation results are presented and discussed in section 5. Our concluding remarks are given in section 6.

\section{SIGNAL MODEL}

Consider an array of $N$ sensors receives $M$ narrow band signals impinging from unknown directions $\boldsymbol{\theta}=\left[\theta_{1}, \ldots, \theta_{M}\right]^{T}$. The array observation vector $\boldsymbol{x}(t) \in \mathbb{C}^{N \times 1}$ can be expressed as

$$
\boldsymbol{x}(t)=\boldsymbol{H}(\boldsymbol{\theta}) \boldsymbol{s}(t)+\boldsymbol{n}(t), \quad t=1,2, \ldots, T
$$

where the $m$ th column of the matrix

$$
\boldsymbol{H}(\boldsymbol{\theta})=\left[\boldsymbol{d}\left(\theta_{1}\right), \ldots, \boldsymbol{d}\left(\theta_{M}\right)\right]
$$

is the steering vector $\boldsymbol{d}\left(\theta_{m}\right) \in \mathbb{C}^{N \times 1}$ associated with the signal arriving from the direction $\theta_{m}$. The signal waveform $\boldsymbol{s}(t)=\left[s_{1}(t), \ldots, s_{M}(t)\right]^{T} \in \mathbb{C}^{M \times 1}$ is considered as deterministic and unknown. Furthermore, the noise vector $\boldsymbol{n}(t) \in \mathbb{C}^{N \times 1}$ is independent, identically complex normally distributed with zero mean and covariance matrix $\nu \boldsymbol{I}$, where 
$\nu$ is the unknown noise spectral parameter and $\boldsymbol{I}$ is an identity matrix with corresponding dimension. Based on the observation $\{\boldsymbol{x}(t)\}_{t=1}^{T}$, our main interest is to determine the number of signals $M$.

\section{SIGNAL DETECTION USING A MULTIPLE HYPOTHESIS TEST}

We suggest a multiple hypothesis test to determine the number of signals. Let $M_{\max }$ denote the maximal number of sources. The following procedure detects one signal after another. More precisely, for $m=1$,

$$
\begin{aligned}
H_{1}: \quad & \text { Data contains only noise. } \\
& \boldsymbol{x}(t)=\boldsymbol{n}(t) \\
A_{1}: \quad & \text { Data contains at least } 1 \text { signals. } \\
& \boldsymbol{x}(t)=\boldsymbol{H}_{1}\left(\boldsymbol{\theta}_{1}\right) \boldsymbol{s}_{1}(t)+\boldsymbol{n}(t)
\end{aligned}
$$

For $m=2, \ldots, M_{\max }$

$$
\begin{aligned}
H_{m}: & \text { Data contains at most }(m-1) \text { signals. } \\
& \boldsymbol{x}(t)=\boldsymbol{H}_{m-1}\left(\boldsymbol{\theta}_{m-1}\right) \boldsymbol{s}_{m-1}(t)+\boldsymbol{n}(t) \\
A_{m}: & \text { Data contains at least } m \text { signals. } \\
& \boldsymbol{x}(t)=\boldsymbol{H}_{m}\left(\boldsymbol{\theta}_{m}\right) \boldsymbol{s}_{m}(t)+\boldsymbol{n}(t)
\end{aligned}
$$

The steering matrix and signal vector are given by $\boldsymbol{H}_{m}\left(\boldsymbol{\theta}_{m}\right)$ $=\left[\boldsymbol{d}\left(\theta_{1}\right), \ldots, \boldsymbol{d}\left(\theta_{m}\right)\right]$ and $\boldsymbol{s}_{m}(t)=\left[s_{1}(t), \ldots, s_{m}(t)\right]^{T}$, respectively.

Based on the likelihood ratio (LR) principle, we obtain the test statistics $T_{m}\left(\hat{\boldsymbol{\theta}}_{m}\right),\left(m=1, \ldots, M_{\max }\right)$ as follows.

$$
\begin{aligned}
T_{m}\left(\hat{\boldsymbol{\theta}}_{m}\right) & =\log \left(\frac{\operatorname{tr}\left[\left(\boldsymbol{I}-\boldsymbol{P}_{m-1}\left(\hat{\boldsymbol{\theta}}_{m-1}\right)\right) \hat{\boldsymbol{R}}\right]}{\operatorname{tr}\left[\left(\boldsymbol{I}-\boldsymbol{P}_{m}\left(\hat{\boldsymbol{\theta}}_{m}\right)\right) \hat{\boldsymbol{R}}\right]}\right) \\
& =\log \left(1+\frac{n_{1}}{n_{2}} F_{m}\left(\hat{\boldsymbol{\theta}}_{m}\right)\right),
\end{aligned}
$$

where $\hat{\boldsymbol{R}}=\frac{1}{T} \sum_{t=1}^{T} \boldsymbol{x}(t) \boldsymbol{x}(t)^{H}$ and $\boldsymbol{P}\left(\hat{\boldsymbol{\theta}}_{m}\right)$ is the projection matrix onto the subspace spanned by the columns of $\boldsymbol{H}_{m}\left(\hat{\boldsymbol{\theta}}_{m}\right)$. When $m=1$, we define $\boldsymbol{P}_{0}(\cdot)=\mathbf{0}$. $\hat{\boldsymbol{\theta}}_{m}$ represents the ML estimate assuming that $m$ signals are present in the observation.

Under hypothesis $H_{m}$, the statistic

$$
F_{m}\left(\hat{\boldsymbol{\theta}}_{m}\right)=\frac{n_{2}}{n_{1}} \frac{\operatorname{tr}\left[\left(\boldsymbol{P}_{m}\left(\hat{\boldsymbol{\theta}}_{m}\right)-\boldsymbol{P}_{m-1}\left(\hat{\boldsymbol{\theta}}_{m-1}\right)\right) \hat{\boldsymbol{R}}\right]}{\operatorname{tr}\left[\left(\boldsymbol{I}-\boldsymbol{P}_{m}\left(\hat{\boldsymbol{\theta}}_{m}\right)\right) \hat{\boldsymbol{R}}\right]}
$$

is $F_{n_{1}, n_{2}}$-distributed. Taking the effect of the nonlinear parameter $\boldsymbol{\theta}_{m}$ into account, the degrees of freedom $n_{1}, n_{2}$ are given by [6]

$$
n_{1}=T\left(2+r_{m}\right), \quad n_{2}=T\left(2 r_{\boldsymbol{x}}-2 m-r_{m-1}\right)
$$

with

$$
r_{\boldsymbol{x}}=\operatorname{dim}(\boldsymbol{x}(t))=N, \quad r_{m}=\operatorname{dim}\left(\boldsymbol{\theta}_{m}\right)=m .
$$

Since $F_{m}\left(\hat{\boldsymbol{\theta}}_{m}\right)^{\prime} s$ distribution depends on $r_{m}$ and $r_{m-1}$, the respective dimension of the parameters $\boldsymbol{\theta}_{m}$ and $\boldsymbol{\theta}_{m-1}$, but not on the true parameter values, it is pivotal. A test statistic is called pivotal if its distribution does not depend on the true value of the parameter.

From eq. (5) it is easy to see that the LR test is equivalent to the F-test proposed by Shumway [7]. The F-test uses $F_{m}\left(\hat{\boldsymbol{\theta}}_{m}\right)$ in testing $H_{m}$ against $A_{m}$. Given $(m-1)$ signals, whether a further signal exists is decided by whether the estimated increase in SNR is large enough.

\section{CONTROL OF FALSE DISCOVERY RATE}

The control of type one error is an important issue in multiple inferences. A type one error occurs when the null hypothesis $H_{m}$ is wrongly rejected. The traditional concern in multiple hypothesis problems has been about controlling the probability of erroneously rejecting any of the true null hypotheses, the familywise error-rate (FWE). Given a certain significance level $\alpha$, the control of FWE requires each of the $M$ tests to be conducted at a lower level. For example, the significance level of each test is given by $\alpha / M$ in the classical Bonferroni procedure. When the number of tests increases, the power of the Bonferroni-type procedures [5] that control the FWE is substantially reduced.

To keep the balance between type one error control and power, Benjamini and Hochberg introduced a new criterion into the multiple comparison problem, the false discovery rate (FDR)[1]. The FDR is defined as the expected proportion of errors among the rejected hypotheses. If all null hypotheses $\left\{H_{1}, H_{2}, \ldots, H_{M}\right\}$ are true, the FDR-controlling procedure controls the traditional FWE. But when many hypotheses are rejected, an erroneous rejection is not as crucial for drawing conclusion from the whole family of tests, the FDR is a desirable error rate to control. In practice, the difference between the FDR- and FWE-controlling procedures becomes more dramatic when the size of the problem is larger [2].

Assume that among the $M$ tested hypotheses $\left\{H_{1}, H_{2}\right.$, $\left.\ldots, H_{M}\right\}, m_{0}$ are true null hypotheses. Let $\left\{p_{1}, p_{2}, \ldots, p_{M}\right\}$ be the $p$-values (observed significance values) corresponding to the test statistics $\left\{T_{1}, T_{2}, \ldots, T_{M}\right\}$. By definition, $p_{m}=1-P_{H_{m}}\left(T_{m}\right)$ where $P_{H_{m}}$ is the distribution function under $H_{m}$. Benjamini and Hochberg showed that when 
(1) the test statistics are independent or (2) the joint distribution of the test statistics is positive regression dependent on the subset of the test statistics corresponding to the true null hypotheses, the following procedure controls the FDR at level $q \cdot m_{0} / M \leq q$ [1] [2].

\section{The Benjamini Hochberg Procedure}

Define

$$
k=\max \left\{m: p_{(m)} \leq \frac{m}{M} q\right\}
$$

and reject $H_{(1)} \ldots H_{(k)}$. When no such $k$ exists, reject no hypothesis.

If the test statistics do not ensure dependency or positive dependency, the above procedure is conducted with $q^{\prime}=q /\left(\sum_{i=1}^{M} \frac{1}{i}\right)$ instead of $q$ in eq. (9) to control the FDR at the same level [2] [4]. Note that using $q^{\prime}$ which is smaller than $q$ will induce a loss in power.

The unmodified Benjamini Hochberg procedure (9) is used in our implementation. Eq. (6) shows that given the estimated noise, $\operatorname{tr}\left[\left(\boldsymbol{I}-\boldsymbol{P}_{m}\left(\hat{\boldsymbol{\theta}}_{m}\right)\right) \hat{\boldsymbol{R}}\right]$, the numerator which is characterized by the difference between the two projection matrices, $\boldsymbol{P}_{m}\left(\hat{\boldsymbol{\theta}}_{m}\right)$ and $\boldsymbol{P}_{m-1}\left(\hat{\boldsymbol{\theta}}_{m-1}\right)$, is independent from the other test statistics. We believe that it is possible to prove independency or positive dependency of the test statistics. Therefore, the unmodified Benjamini Hochberg procedure should be able to control the FDR in the suggested multiple test.

\section{SIMULATION}

We test the proposed algorithms by numerical experiments. The narrow band signals generated by sources of different strengths are received by a uniformly linear array of 15 sensors with inter-element spacings of half a wavelength. Each experiment performs 200 trials. The number of snapshots is given by $T=15,50$. For comparison, the simulated data is applied to the Bonferroni-Holm procedure [5] as well. The sequentially rejective Bonferroni-Holm procedure keeps the FWE at the same level $\alpha$ as the classical Bonferroni test but is more powerful than it. The significance level of each test is given by $\alpha /(M+1-m)$. We use $q=0.05$ and $\alpha=0.05$ in the simulation.

In the first experiment, we consider three sources located at $\boldsymbol{\theta}=\left[\begin{array}{lll}12^{\circ} 36^{\circ} & 45^{\circ}\end{array}\right]$. The SNR, which is defined as $10 \log \left(s_{i}(t)^{2} / \nu\right), i=1, \ldots, m$, varies from -15 to $10 \mathrm{~dB}$ in a $1 \mathrm{~dB}$ step. The SNR differences $=\left[\begin{array}{lll}-2 & 0 & 1\end{array}\right]$ dB. Fig. 1 shows the number of detected sources averaged over 200 trials vs SNR. In general, more signals are detected at higher SNRs. The FDR-controlling procedure has an overall higher number of detected signals than the FWEcontrolling procedure. While both algorithms behave almost identically at $T=50$, the FDR-controlling procedure performs slightly better at $T=15$.

In the second experiment, the signals are generated by 6 sources located at $\boldsymbol{\theta}=\left[\begin{array}{llll}12^{\circ} & 21^{\circ} 36^{\circ} 45^{\circ} 56^{\circ} 64^{\circ}\end{array}\right]$ with SNR differences $=\left[\begin{array}{llllll}-2 & 0 & 0 & 1 & 2 & -1\end{array}\right]$ dB. From fig. 2 we can observe that the Benjamini-Hochberg procedure always detects more signals than the Bonferroni-Holm procedure. The Benjamini-Hochberg procedure has a larger gain in power at $T=15$ than at $T=50$.

In the third experiment, we consider $M=12$ signals. The SNR varies from -30 to $20 \mathrm{~dB}$ in a $2 \mathrm{~dB}$ step. Results obtained from $T=15$ and $T=50$ are presented in figs. 3 and 4, respectively. The FDR-controlling procedure always outperforms the FWE-controlling procedure. In the low SNR region, -30 to $-10 \mathrm{~dB}$, the difference between these two approaches is larger than in the high SNR region.

Comparing the three experiments, we conclude that the FDR- and FWE-controlling procedures have a similar performance when the number of signals is small but differ from each other when the number of signals increases. As predicted in the theory, the FDR-controlling procedure is preferable to the FWE-controlling procedure as the size of the problem grows.

\section{CONCLUSION}

This work discusses signal detection using a multiple hypothesis test under an FDR consideration of Benjamini and Hochberg. Compared to the classical FWE criterion, the FDR criterion leads to more powerful tests and controls the errors at a reasonable level. We expect that average overestimation of the number of signals would be improved by the FDR-controlling procedure because it is designed to do so. Simulation shows that the proposed multiple hypothesis test provides good results at low SNRs and small numbers of snapshots. The FDR-controlling approach performs better than the FWE-controlling approach. Distinction between these two approaches becomes significant for a large number of signals and low SNRs. We will provide additional examples and details in a future publication.

\section{REFERENCES}

[1] Y. Benjamini and Y. Hochberg. Controlling the false discovery rate: a practical and powerful approach to multiple testing. J. Roy. Statist. Soc. Ser. B, (57):289300, 1995.

[2] Y. Benjamini and D. Yekutieli. The control of the false discovery rate in multiple testing under dependency. Ann. Statist., (4):1165-1188, 2001. 


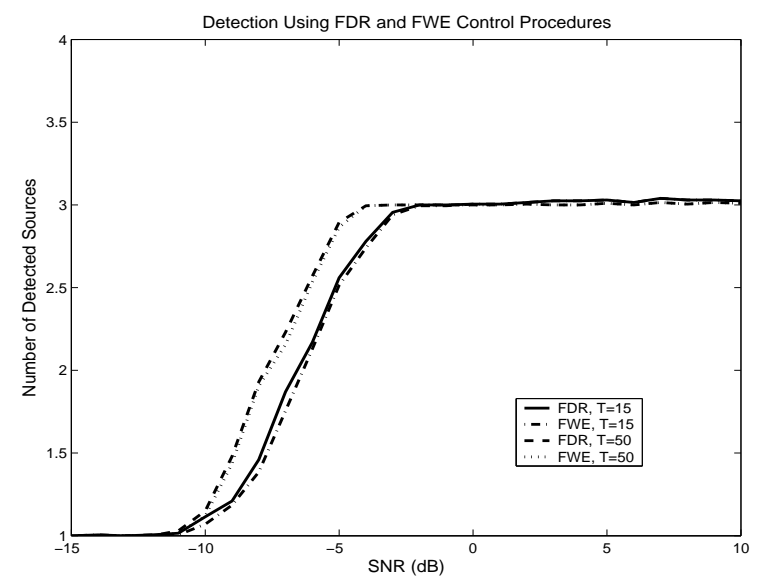

Fig. 1. Number of detected signals. $\boldsymbol{\theta}=\left[12^{\circ} 36^{\circ} 45^{\circ}\right]$, SNR $=[-15$ : $1: 10] \mathrm{dB}$, SNR difference $=\left[\begin{array}{lll}-2 & 0 & 1\end{array}\right] \mathrm{dB}$, number of snapshots $T=$ 15,50 .

[3] Johann F. Böhme. Statistical array signal processing of measured sonar and seismic data. In Proc. SPIE 2563 Advanced Signal Processing Algorithms, pages 2-20, San Diego, Jul 1995.

[4] C. R. Genovese, N. A. Lazar, and T. Nichols. Thresholding of statistical maps in functional neuroimaging using the false discovery rate. NeuroImage, 15:870$878,2002$.

[5] S. Holm. A simple sequentially rejective multiple test procedure. Scand. J. Statist., 6:65-70, 1979.

[6] D. Maiwald. Breitbandverfahren zur Signalentdeckung und -ortung mit Sensorgruppen in Seismik- und Sonaranwendungen. Dr.-Ing. Dissertation, Dept. of Electrical Engineering, Ruhr-Universität Bochum, Shaker Verlag, Aachen, 1995.

[7] R.H. Shumway. Replicated time-series regression: An approach to signal estimation and detection. In D.R. Brillinger and P.R. Krishnaiah, editors, Handbook of Statistics, Vol. 3, pages 383-408. Elsevier Science Publishers B.V., 1983.

[8] M. Wax and T. Kailath. Detection of signals by information theoretic criteria. IEEE ASSP, 33(2):387-392, April 1983.

[9] M. Wax and I. Ziskind. Detection of the number of coherent signals by the mdl principle. IEEE ASSP, 37(8):1190-1196, August 1989.

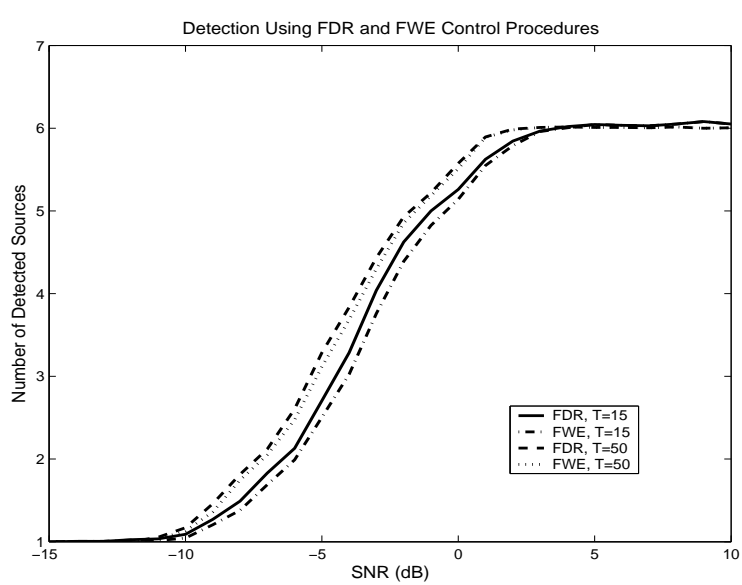

Fig. 2. Number of detected signals. $\boldsymbol{\theta}=\left[12^{\circ} 21^{\circ} 36^{\circ} 45^{\circ} 56^{\circ} 64^{\circ}\right]$, $\mathrm{SNR}=\left[\begin{array}{ll}-15: 1: 10\end{array}\right] \mathrm{dB}$, SNR differences $=\left[\begin{array}{llllll}-2 & 0 & 0 & 1 & 2 & -1\end{array}\right] \mathrm{dB}$, number of snapshots $T=15,50$.

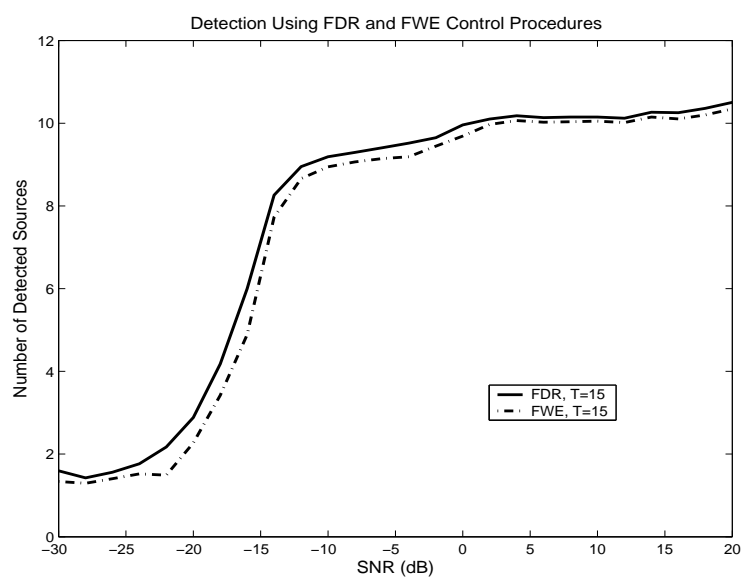

Fig. 3. Number of detected signals. $M=12, \mathrm{SNR}=[-30: 2: 20]$ $\mathrm{dB}$, number of snapshots $T=15$.

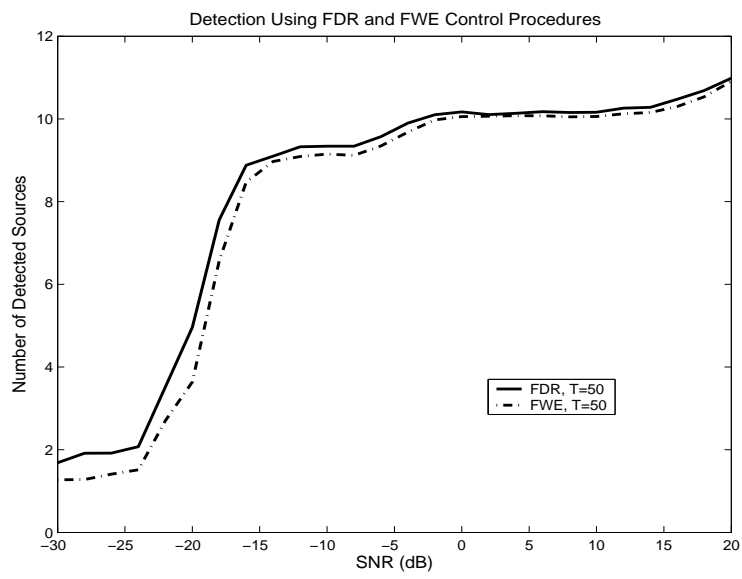

Fig. 4. Number of detected signals. $M=12, \mathrm{SNR}=[-30: 2: 20]$ $\mathrm{dB}$, number of snapshots $T=50$. 